\title{
An analytic-geospatial approach for sustainable water resource management: a case study in the province of Perugia
}

\author{
Stefano Casadei, ${ }^{1}$ Michele Bellezza, ${ }^{2}$ Luca Casagrande, ${ }^{2}$ Arnaldo Pierleoni ${ }^{2}$ \\ ${ }^{1}$ Department of Civil and Environmental Engineering, University of Perugia, Italy; \\ ${ }^{2}$ T4E S.r.I. one Technology four Elements, Spin-Off UNIPG, Perugia, Italy
}

\begin{abstract}
Water is a strategic, but also highly vulnerable, natural resource. This because the increasing demand from multiple uses, in many cases competing amongst them, seems to influence the concepts of sustainability of the exploitation. From the operational point of view, the studied system is an integrated decision support system. It is not only a platform to exchange information and assessments, but also a tool for conflict resolution, in the management of water resources, to obtain the consensus among all participants in the decisional processes. So the canonical "top-down" approach has been replaced with a "bottom-up" approach where all stakeholders become decision makers themselves. The application of the aforementioned approach was studied for the Tiber River basin and has been applied to the Province of Perugia area. The study focused to the building of a spatial database of hydrological data and multipurpose water withdrawals, together with the setting of the evaluation model for the surface water resources. This model bases its algorithms on regionalization procedures of flow parameters. For the definition of the river condition, hydrological indices calculated from the hydrological database have been used, while for the existing withdrawals, an analysis procedure has been developed, that from the point of interest directly selected on the map, finds out the upstream basin and, by means of overlay procedures, identifies the upstream water uses and the total flow that could be extracted. The potential of the system and the technologies used are contained in a WEB platform that allows the analysis of the database of water uses/withdrawals on the cartography, and the comparison with the hydrogeological characteristics of the sub-basin examined.
\end{abstract}

\footnotetext{
Correspondence: Stefano Casadei, Department of Civil and Environmental Engineering, University of Perugia, Borgo XX Giugno 74, 06121 Perugia, Italy.

Tel. +39.075.5856043 - Fax: +39.075 .5856049 .

E-mail: casadei@unipg.it
}

Key words: water resource, decision support system, WebGIS

Acknowledgments: this research is part of the project defined and funded by the Province of Perugia - Division of Defence and Management Hydraulics

(C) Copyright S. Casadei et al., 2013

Licensee PAGEPress, Italy

Journal of Agricultural Engineering 2013; XLIV(s2):e163

doi:10.4081/jae.2013.s2.e163

This article is distributed under the terms of the Creative Commons Attribution Noncommercial License (by-nc 3.0) which permits any noncommercial use, distribution, and reproduction in any medium, provided the original author(s) and source are credited.
The purpose of this study is to provide software tools that can be used as a support in water resource evaluation and management policies at the basin scale.

\section{Introduction}

The new approach in water resources management is based on the building of a spatial database of hydrological data and multipurpose water withdrawals, together with the setting of the evaluation model for the surface water resources.

This model bases its algorithms on regionalization procedures of flow parameters derived from the geomorphologic features of the basin, Area and Base Flow Index (BFI) (Casadei, 1995). The output is a set of Flow Duration Curves (FDCs) for each arc of the simplified network (Vogel and Fennessy, 1995). For the definition of the river condition, hydrological indices such as BFI, $Q_{7,10}, Q_{355}, Q_{347}$, entire FDC are used (Singh and Stall, 1974; Smakhtin, 2001). They are calculated from the hydrological database, while for the existing withdrawals an analysis procedure has been developed, that from the point of interest directly selected on the map, finds out the upstream basin and, by means of overlay procedures, identifies the upstream water uses and the total flow that could be extracted.

In this work hydrological topics are linked with the Information and Communication Technologies (ICT) for environmental sustainability. The use of the Web Processing Service (WPS) protocol makes possible to perform the typical operation of Desktop GIS application, through a Web interface and via an HTTP protocol. The main procedures require as search parameters both numerical inputs (such as the kind of water use, the maximum allowed withdrawal, etc) and geographical (such as withdrawals upstream of a certain section of the network). For this specific kind of search it is necessary to use the potentialities of a GIS software since it implies the spatial analysis of the river network, together with a database containing the information of the basin. Therefore the system is composed of a relational database with geospatial extension for vectorial data; the GIS engine that performs all the geospatial operations and finally the PyWPS that is a Python implementation of the WPS protocol. The WEB interface offers various thematisms in order to provide a better support to the decisional process. The thematisms are shown by means of a the Web Map Service protocol taking advantage of the javascript framework OpenLayers. The project is entirely based on Open Source Technology (Neteler and Mitasova, 2008).

\section{Materials and methods}

\section{Data analysis and modeling processes}

The first step has been the creation of a shared database at the 
basin scale (Tiber River) of all data and information together with user friendly procedures for its periodical update.

This final goal has been achieved by a software platform that could allow a free sharing of information, which is provided by a WEB-BASED architecture.

The creation of the database leads to the developing of a series of procedures concerning the analysis of the data. These procedures can be both numerical and graphical, according to the typology of the data and the kind of analysis concerned.

The computational side of the data analysis also foresees the possibility of performing statistical evaluations, and in this field there is a wide range of possibilities (Katz et al., 2002) that perform nicely in a WEB-GIS environment.

At present, the procedures implemented are aimed towards the definition of various hydrological indexes for defining the base flow value in low-flow conditions in order to be able to correctly assess the water resources and/or the available amount in a section of a network, either in order to issue or renew a withdrawal license or to study the state of the watercourse.

\section{BFI calculation}

The analysis of hydrographs of daily flow is a useful technique in a wide variety of studies in the field of water resources. In particular, the breakdown of flow rates in the two components of base flow and surface runoff is often used to identify contributions from springs and groundwater (Sloto et al., 1991). In this context, it is now definitely clear that there is a close relation between the geology of the basin and the base flow.

The hydrogeological features of a basin (intensity limit infiltration, effective porosity, permeability of rock formations, etc.), directly affect low flow regimes of a stream. It is therefore of great interest to establish a quantitative relationship between base flow and these features. Unfortunately it is difficult to summarize in a single parameter the complex hydrological features mentioned above, unless we assume as a measure of these characteristics the effects that they produce, i.e. the base flow itself.

From an operative point of view, the BFI calculation (Figure1) is made according to Lvovich method (Lvovich, 1972). The index is the ratio between the volume of base flow, area under yellow line, and the total volume, area under the total hydrograph (blue line). The yellow line is obtained by means of a procedure of data analysis conducted on

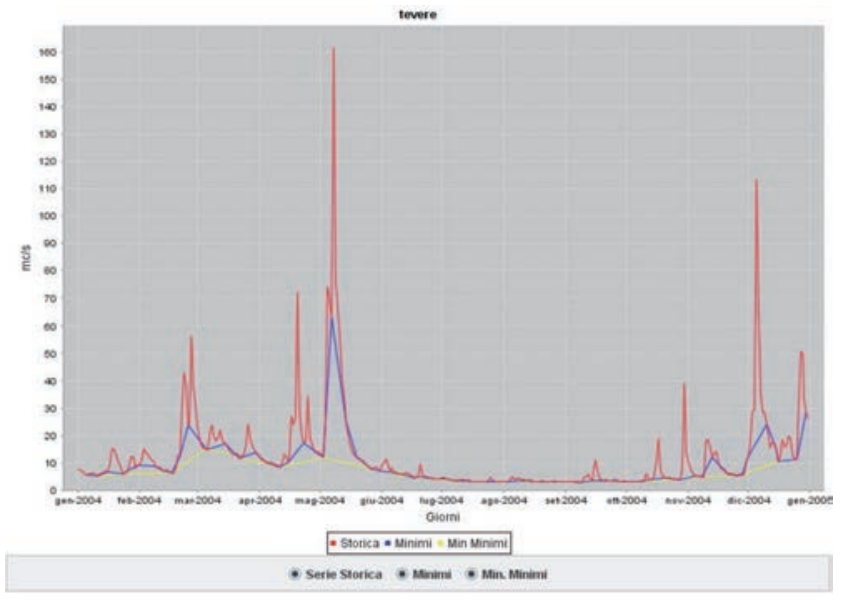

Figure 1. Example of applet view of BFI calculation by measured data at S. Lucia station, Tiber River. the minimum flow of 5oups, then processed in groups of three values in a moving average and finally filtered according to the following conditions: $0.9 \mathrm{Qmin}(\mathrm{I}) \leq \mathrm{Qmin}(\mathrm{I}+1)$ and $0.9 \mathrm{Qmin}(\mathrm{I}) \leq \mathrm{Qmin}(\mathrm{I}-1)$, for $\mathrm{I}=2, \mathrm{~N}-1$.

The BFI of the basin is the average of the time series of annual BFI, but it is also possible elaborate the consecutive time series of flow data.

As mentioned, the index of the base flow is closely related to the nature of the hydrogeological formations in the basin. Indeed with increasing occurrence of permeable formations there is a direct variation of the BFI calculated on the basis of observed data, while an increase in the occurrence of impermeable formations inverts the relation (Manciola and Casadei, 1991) .

The BFI of the ungauged basin is thus obtained using a weighted average method, compared to areas, with BFI typical of single formations, assuming that every formation has a specific value of BFI which is evaluated in the gauged basins:

$$
\mathrm{BFI}_{\mathrm{est}, \mathrm{j}}=\sum_{\mathrm{i}=\mathrm{n}}^{\mathrm{n}} \frac{\mathrm{A}_{\mathrm{j}, \mathrm{i}}}{\mathrm{A}_{\mathrm{j}, \mathrm{tot}}} \cdot \mathrm{BFI}_{\mathrm{fm}, \mathrm{i}}
$$

Where:

$\mathrm{A}_{\mathrm{j}, \mathrm{i}}=$ superficial extension of the $\mathrm{i}^{\text {th }}$ hydrogeological formation in the $\mathrm{j}^{\text {th }}$ basin

$\mathrm{A}_{\mathrm{j}, \mathrm{tot}}=$ total area $\mathrm{j}^{\text {th }}$ basin

$\mathrm{BFI}_{\text {est, }}=$ extimated BFI in the $\mathrm{j}^{\text {th }}$ basin

$\mathrm{BFI}_{\mathrm{frm}, \mathrm{i}}=\mathrm{BFI}$ associated to the $\mathrm{i}^{\text {th }}$ hydrogeological formation

This last term has been evaluated in the studied area, where every hydrogeological formation has been linked to a value of BFI by means of a technique which minimizes the estimation error in the gauged station (Manciola and Casadei, 1991).

\section{$Q_{\mathrm{n}, \mathrm{m}}$ calculation}

The value of $Q_{n, m}$ describes the low flow characteristics of the river, represented by the series of annual minimum flow averaged over an interval of $n$ consecutive days with a return time of $m$ years. This index is implemented by various US Agencies for environmental protection, that generally adopt the $Q_{7,10}$ value.

For the calculation of $Q_{n, m}$ a series of steps that begin with the calculation of minimum average daily flow over n-days are required. This calculation is performed for each year of the series. From these values the average and variance are calculated in order to evaluate the parameters of the Weibull-Gumbel distribution:

$$
\mathrm{P}(\mathrm{x})=1-\exp \left[-\left(\frac{\mathrm{x}-\varepsilon}{\theta-\varepsilon}\right)^{1 / \lambda}\right]
$$

Where:

$\varepsilon=$ lower limit of the distribution

$\lambda=$ shape parameter

$\theta=$ scale parameter

So, in order to produce estimates of the parameters of the distribution, more procedures have been implemented, especially for the estimation of the parameter $\lambda$, from which also derives the parameter $\varepsilon$, and this one is essential to locate the minimum value of the distribution itself (Manciola et al., 2004).

The regionalization process of the minimum flow of 7 days with return time of 10 years $\left(Q_{7,10}\right)$ is based on the study of regressions between calculated data and hydrogeological parameters of the gauged basin. From a methodological point of view an estimate of the $Q_{7,10}$ that can fit the entire basin is still to be made, instead estimates for different portions of it, identifying one or more relations like the following:

$\frac{\mathrm{Q}_{7,10}}{\mathrm{~A}_{\mathrm{tot}}}=\sum_{\mathrm{k}=1}^{3} \mathrm{a}_{\mathrm{k}} \cdot \mathrm{BFI}^{\mathrm{k}}+\mathrm{a}_{0}$ 
where the constants are taken from the interpolation procedure conducted starting from the values of calculated $Q_{7,10}$, possibly on natural and naturalized flow values (i.e. not influenced by human factors). The methodological approach does not vary with the change of the characteristics of the index in terms of $n$ and $m$, it should be noted that the polynomial estimate does not exceed in any case the third order (Ubertini et al, 1996) .

\section{Flow duration curves}

The FDCs are meant as a synthesis of the hydrological status, before and after the human modifications, of each section of the river network.

The use of FDC as a tool for assessing available water resources is possible by means of regionalization procedures, using characteristic parameters of the basin, automatically calculated by querying the GIS engine that performs the analysis of the geomorphology of the basin (BFI and Area, see Bellezza et al., 2007).

In this case, the main river network is divided into sections for which it is possible to know all relevant information that influence the availability of water. These data, through the theoretical regionalization procedure, allow the determination of the hypothetical natural flow for each section (Figure 2).

This information can be evaluated in terms of low flow indexes, i.e. Q 355-days and 347-days duration, or overlapped to the minimum instream flow for this section (DMV), if defined.

However, this information cannot be considered enough for the management of the resource at the scale of the basin concerned, in fact it is necessary to overlay the hydrological and hydrogeological information that quantifies the natural resources available, with the information about all the licences of water withdrawal.

\section{Water withdrawals management}

This step, of course, implies an expansion of the database with the information about water withdrawals. These data are from different administrations and authorities and are highly non- homogeneous and incomplete to the point that the recent legislation has many times imposed a reorganization of the public water resources dataset (Ministry of the Environment, 2004). Achieving this result can surely be made eas- ier through the creation of a unified database of withdrawals at the basin scale. This database should also be made accessible to the several administrations concerned via a WEB portal where they can operate or modify, update present data and insert new ones. Indeed, we think that sharing a single database is the only effective way to reorganize this strategic sector of the water resources management.

For this reason, the main technologies used within the system are contained in the section of the portal that allows the analysis of the database of water uses/withdrawals on the cartography. This is probably the most significant section, in terms of low flow management, because the search can easily point out the situation, even at the subbasin scale, and make a comparison with the hydrogeological features of the sub-basin examined, that are particularly influential especially during droughts, also making easily understandable the spatial distribution of licences and their information.

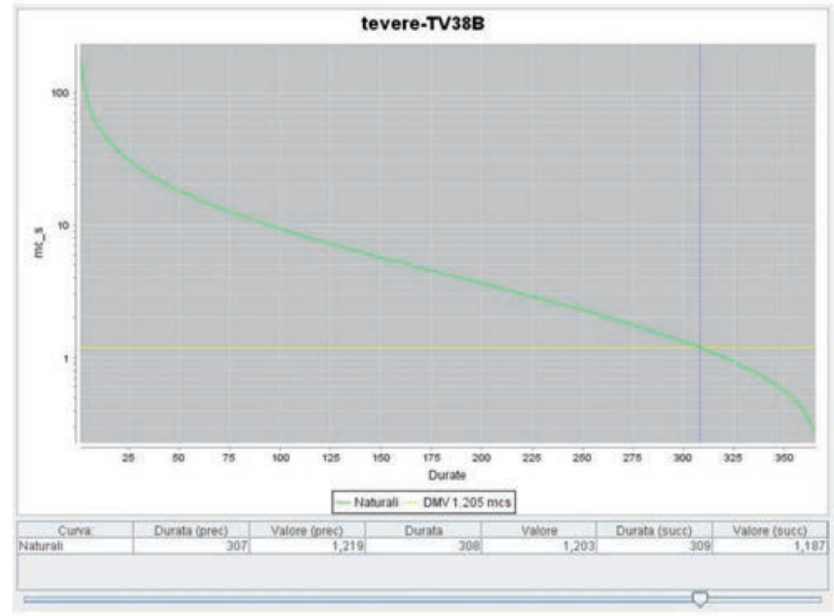

Figure 2. Example of applet view of flow duration curve.

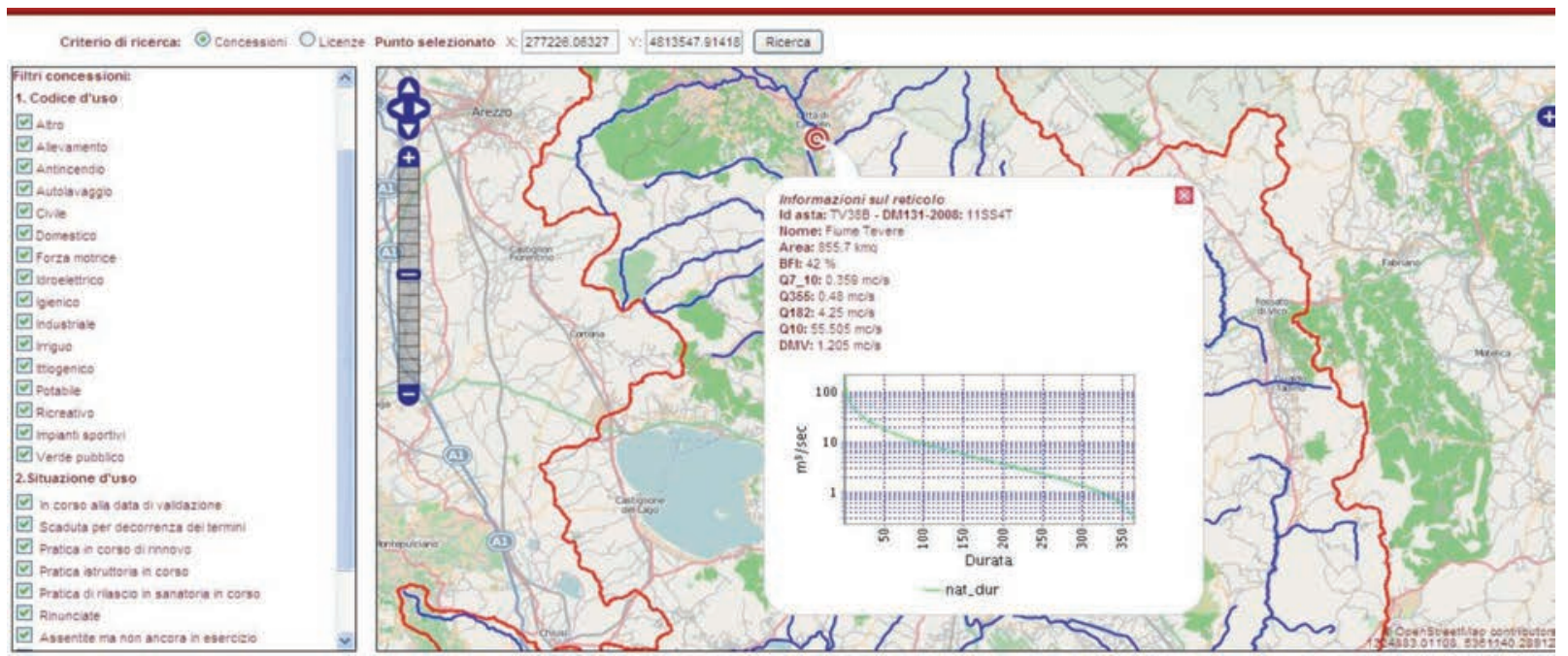

Figure 3. Search example of the hydrological status in a sub-basin. 
In order to evaluate the river conditions the previous hydrological indices were used (Figure 3), while for the existing water withdrawals an analysis procedure has been developed, that from the point of interest selected directly on the map, finds out the upstream basin and, by means of overlay procedures, identifies the upstream water uses which are geo-referenced on the map. To search the water body in more details, a filtered search has been developed so that the uses can be retrieved on the base of their type, the allowed amount of water, and their administrative status (Figure 4).

\section{Results and discussion}

The development of this study shows the greatest difficulty in the fragmentation of responsibilities among administrations located in succession along the river network, this is why administrations downstream do not know the upstream situation (water availability), while those upstream are not able to evaluate the amount of water withdrawn downstream. At the moment this situation generates a stalemate, for this reason a web tool operating on a single database of licences, that should be constantly kept updated by all the administrations involved, can be very useful.

This is the reason why the initial case study has been the total Tiber River Basin, which has $17462 \mathrm{Km}^{2}$ of area distributed over 6 Regions and 8 main Province. The second step has been an in depth analysis the area managed by the Province of Perugia.

In order to make the technical-administrative side of the management of the superficial water resource in a much easier and immediate way, a web site (Province of Perugia, 2012) has been created. In this portal all the methodologies described above are becoming operative and accessible.

The data collection and validation phase brought to the compilation of a hydrological database, already accessible online, that has different levels of access according to the typology of user and the kind of information required. It is made of about 11800 years of hydro-meteorological data and about 10000 information regarding the uses and the water withdrawal licenses. The data can be viewed both in a numeric format and a graphic visualization and if needed, easily exportable in various file formats.
The main result obtained in this study is in demonstrating that it is technically possible to conduct a spatial analysis, at the level of the basin or sub-basin, between the use of surface water resources and the availability of water in low flow condition. The final data show some nobalanced conditions, and many limit conditions, demonstrating the bad water management politics in the last decades.

Therefore, this situation has directed the study toward finding solutions, which using the same tool for spatial analysis, could propose the use of non-conventional water resources.

These analysis also highlighted the presence of a large number of small reservoirs, especially in the Apennines area, whose data were available in the Province of Perugia. So, the same application was extended in order to include data regarding small reservoirs, with the aim of evaluating the possibility of exploitation of this resource that is usually almost completely neglected, even during dry periods.

The results were very encouraging, in fact the possible use of small reservoirs for irrigation can contribute in many cases to restore the equilibrium in the water balance (Figure 5).

In addition, this result indicates that the application can also be extended to the possible use of well water or other available water resources in the area, but only when data are properly recorded and cataloged.

\section{Conclusions}

The purpose of this study is to provide software tools that can serve as a support in water resource evaluation and management policies at the basin scale, with particular attention given to the creation of a database of uniform data that can be easily updated, and to the development of mathematical models that are easy to use, both as regards the interpretation of output data and the choosing of management hypotheses.

The database of water withdrawals, with its related tools, is one of the most significant parts of the work, and if adopted, it will allow to define a more accurate scenario of water use at the basin level. This result may highlight imbalances and failures, and then point out the need for other water resources. The latter may be identified, for example, in small reservoirs, wells, wastewater reuse, and in any case will be handled by the system as geospatial data in the water balance of the basin.

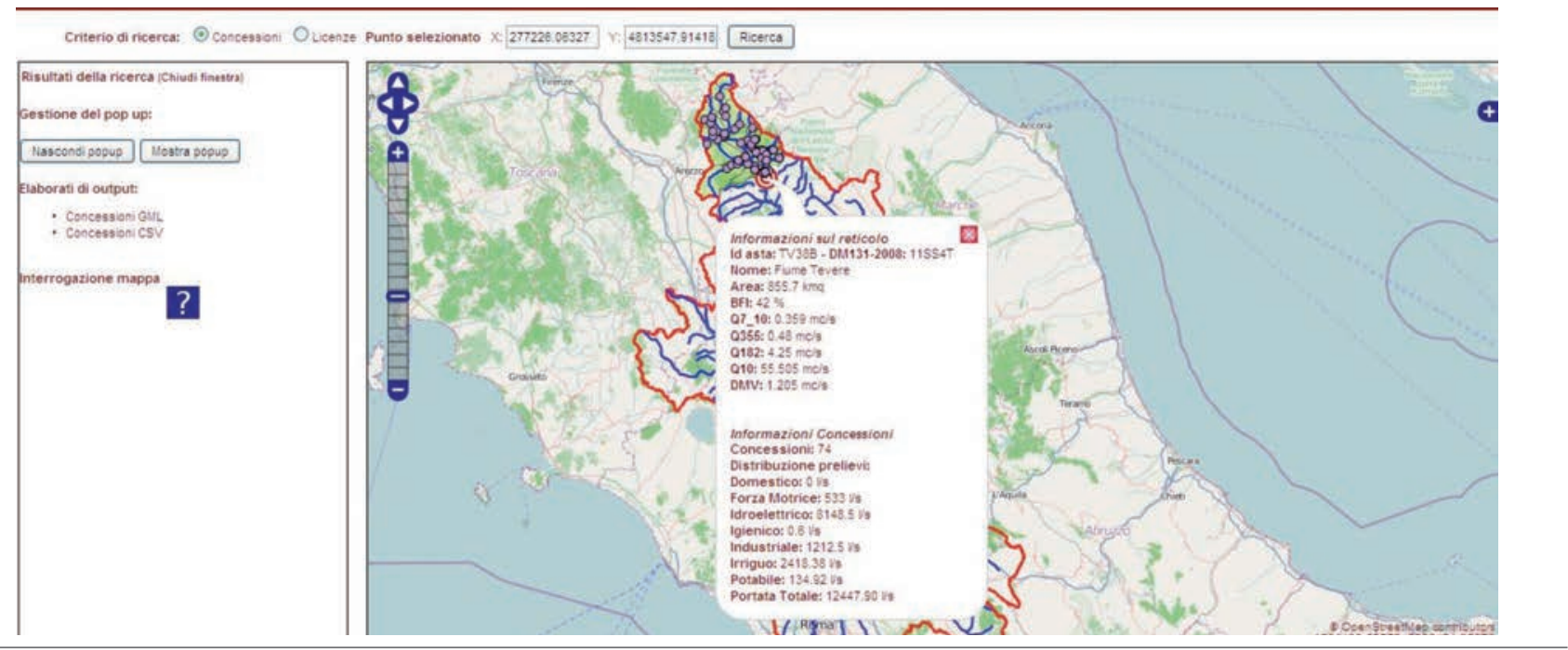

Figure 4. Search example of water withdrawals in a sub-basin versus low flow indeces. 


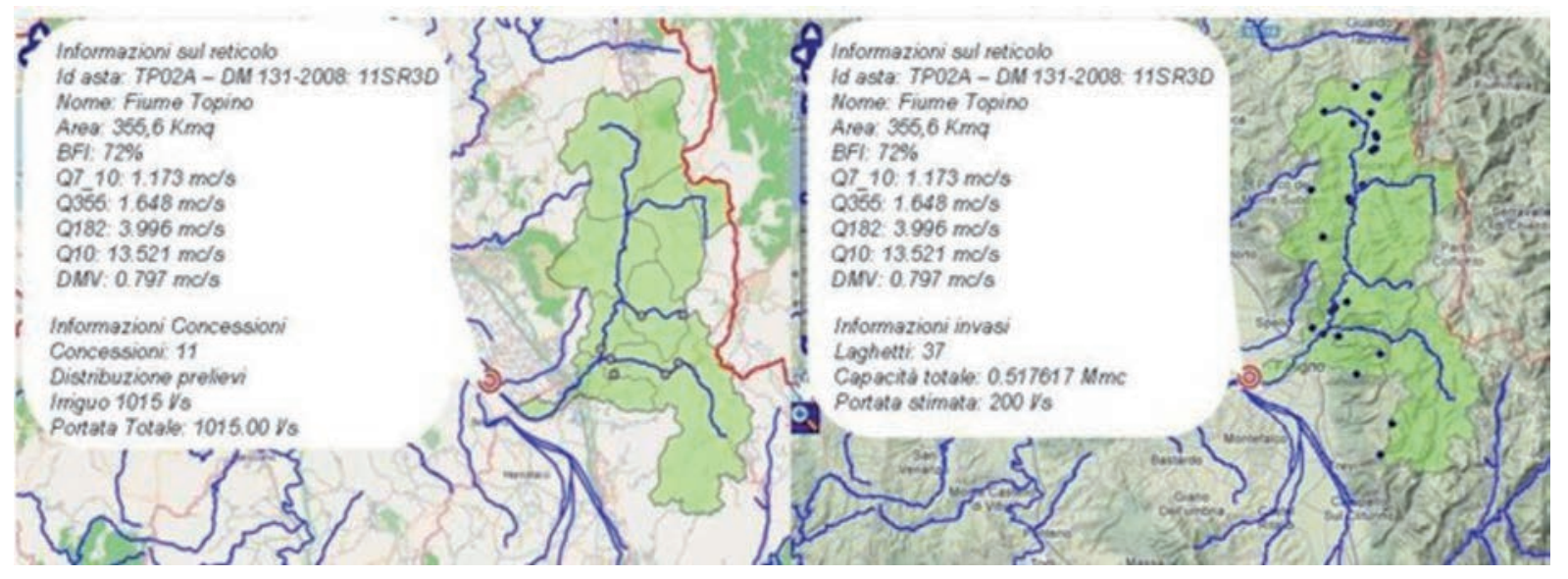

Figure 5. Example of limit condition in water resources management (A) and available water integration from small reservoir (B).

Because of this, the ICT approach and the WebGIS platform represent the added value of the project. The use of algorithmic geospatial analysis within the common GIS software, needs a certain know-how ranging from the use of different data formats to the concept of reference systems, but the overall system is high-performance.

The case study carried on in the Province of Perugia confirms the good performance of the results of the research in the practical field, in particular all the problems underlying the concept of water withdrawal must be assessed carefully, taking into account the existing withdrawals and hydrological situation of the basin concerned.

Future developments cannot only concern techno-scientific investigations, but they will need more information about water resource data management. This problem will involve Institutions and all the stakeholders taking part in the decisional process. This means that only a real and complete sharing of the tool amongst all the participants in the managing process will allow further development in the study and research presented in this paper.

\section{References}

Bellezza M., Casadei S., Manciola, P., Pierleoni, A. 2007. Mathematical model for multipurpose water resource management. In: Proc. 22nd European Regional Conference ICID-CIID ERWG Water Resources Management and Irrigation and Drainage Systems Development in the European Environment, Pavia, Italy.

Casadei S. 1995. Analisi dell'indice del deflusso di base (BFI) di alcuni bacini dell'Italia Centrale. Idrotecnica 3: 177-191.

Katz R. W., Parlange M. B., Naveau P. 2002. Statistics of extremes in hydrology. Adv. Water Resour. 25: 1287-1304.
Lvovich M.I. 1972. Hydrologic budget of continents and estimate of the balance of global fresh water resources. Sov. Hydrol. 4: 360-439.

Manciola P., Casadei S. 1991. Low flow index and hydrogeological characteristics. Proc. of the National Conference on Hydraulic Engineering, ASCE, Nashville, July 29-August 2: 930-936.

Manciola P., Pierleoni A., Bellezza M., Casadei S. 2004. Weibull distribution parameters computation in low flow phenomena. Proc. of the IASTED International Conference on Environmental Modelling and Simulation, St. Thomas, US Virgin Island: 192-196.

Ministry of the Environment, 2004. Guidelines for the preparation of the basin water balance and criteria for the water withdrawals evaluation and for the definition of minimum instream flow, art. 22, paragraph 4, of Legislative Decree No. 152 of 11 May 1999. In: Official Journal No. 268, 15/11/2004.

Neteler M., Mitasova H. 2008. Open source GIS: a GRASS GIS approach. 2nd ed., Springer.

Province of Perugia, 2012. Available from: http://demo.t4e.it/pivrid/

Singh K.P., Stall I.B. 1974. Hydrology of 7-day 10-yr low flow. J. Hydraul. ENG-ASCE, 100, 1753-1771.

Sloto R.A., 1991. A computer method for estimating ground-water contribution to streamflow using hydrograph-separation techniques. In: U.S. Geological Survey Water-Resources Investigations. Report 90-4162: 101-110.

Smakhtin V. U. 2001. Low flow hydrology: a review. J. Hydrol. 240, 147186.

Ubertini L., Manciola L., Casadei S. 1996. Evaluation of the minimum instream flow of the Tiber river basin. Environmental Monitoring and Assessment, vol. 41, n.2, 125-136.

Vogel R. M. and Fennessy N. M. 1995. Flow duration curve II: a review of application in water resources planning. Water Resour. Bull. 31,6, 1029-1039. 